1.1. Letters to and from Abraham Ortelius

\begin{tabular}{|c|c|c|c|c|}
\hline Hessels & Date & From & To & Autograph letter \\
\hline 6 & [1556] 0408 & Abraham Ortelius & Emanuel van Meteren & Brussel, KB, MS III 936, nr. 1 \\
\hline 7 & [1557] 1025 & Abraham Ortelius & Emanuel van Meteren & New York (NY), Pierpont Morgan Library, LHMS, Unbound International Ortelius, MA 2633 \\
\hline 8 & [1559] 0703 & Abraham Ortelius & Emanuel van Meteren & Amsterdam, UBA, Hs. Ge 4a \\
\hline 9 & [1559] 0925 & Abraham Ortelius & Emanuel van Meteren & Brussel, KB, MS III 936, nr. 2 \\
\hline 10 & 15610615 & Joannes Terenumus (Vryfpenninck) & Abraham Ortelius & Sotheby's 1968, lot 391: buyer Nico Israel (Amsterdam) \\
\hline 11 & 15610616 & Scipio Fabius & Abraham Ortelius & Austin (Texas), UL, HRHRC Collection, HRC 55 \\
\hline 13 & 15630922 & Joannes Sambucus & Abraham Ortelius & Cambridge (Mass.), Harvard University, Houghton Library, MS Lat. 225 \\
\hline 14 & 15640619 & Joannes Sambucus & Abraham Ortelius & Cambridge (Mass.), Harvard University, Houghton Library, MS Lat. 225 \\
\hline 15 & 15650414 & Scipio Fabius & Abraham Ortelius & Austin (Texas), UL, HRHRC Collection, HRC 55 \\
\hline 16 & 15660508 & Guido Laurinus & Abraham Ortelius & Den Haag, KB, MS 79 C 4 (002), fol. 123 \\
\hline 17 & 15660827 & Abraham Ortelius & Emanuel van Meteren & Amsterdam, UBA, Hs. Ge $4 \mathrm{~b}$ \\
\hline 18 & 15670225 & Justus Laureins [= Guido Laurinus?] & Abraham Ortelius & Den Haag, KB, MS 79 C 4 (003), fol. 124 \\
\hline 19 & 15670409 & Guillaume Postel & Abraham Ortelius & Den Haag, KB, MS 79 C 4 (005), fol. 174-175 \\
\hline 20 & 15670424 & Guillaume Postel & Abraham Ortelius & Amsterdam, UBA, Hs. Gm 4 \\
\hline 21 & 15670518 & Joannes Thorius senior & Abraham Ortelius & Den Haag, KB, MS 79 C 4 (006), fol. 223 \\
\hline 22 & 15670614 & Joannes Thorius senior & Abraham Ortelius & Den Haag, KB, MS 79 C 4 (007), fol. 224 \\
\hline 23 & 15671213 & Abraham Ortelius & Emanuel van Meteren & Brussel, KB, MS III 936, nr. 3 \\
\hline 24 & 15680305 & Joannes Radermacher senior & Abraham Ortelius & Den Haag, KB, MS 79 C 4 (018), fol. 182-183 \\
\hline 25 & 15680621 & Jean de Vendeville & Abraham Ortelius & Austin (Texas), UL, HRHRC Collection, HRC 55 \\
\hline 26 & 15680712 & Joannes Thorius senior & Abraham Ortelius & Den Haag, KB, MS 79 C 4 (008), fol. 225 \\
\hline 27 & 15680803 & Humphrey Lhuyd & Abraham Ortelius & Aberystwyth, NLW, MS 13187E \\
\hline 28 & 15700302 & Hubertus Goltzius & Abraham Ortelius & Den Haag, KB, MS 79 C 4 (020), fol. 100 \\
\hline 29 & 15700812 & Jacobus Navarchus & Abraham Ortelius & Den Haag, KB, MS 79 C 4 (023), fol. 163 \\
\hline 30 & 15701030 & Johann Crato von Crafftheim & Abraham Ortelius & Den Haag, KB, MS 79 C 4 (024), fol. 60 \\
\hline 31 & 15701102 & Robert Owen & Abraham Ortelius & Aberystwyth, NLW, MS 13187E \\
\hline 32 & 15701122 & Gerardus Mercator & Abraham Ortelius & Austin (Texas), UL, HRHRC Collection, HRC 55 \\
\hline 33 & {$\left[\begin{array}{llllllllllll}1570 & 00 & 00\end{array}\right]$} & Pietro Bizari & Abraham Ortelius & Austin (Texas), UL, HRHRC Collection, HRC 55 \\
\hline 34 & {$[1571] 0102$} & Hugo Owen & Abraham Ortelius & Aberystwyth, NLW, MS 13187E \\
\hline 35 & 15710301 & Joannes Neodicus & Abraham Ortelius & Den Haag, KB, MS 79 C 4 (029), fol. 164 \\
\hline 36 & 15710930 & Hieronymus de Rhoda & Abraham Ortelius & Den Haag, KB, MS 79 C 4 (030), fol. 193-194 \\
\hline 37 & 15720206 & Georgius Braun & Abraham Ortelius & Den Haag, KB, MS 79 C 4 (031), fol. 19-22 \\
\hline 38 & 15720509 & Gerardus Mercator & Abraham Ortelius & Austin (Texas), UL, HRHRC Collection, HRC 55 \\
\hline 39 & 15720515 & Cesare Orlandi & Abraham Ortelius & Austin (Texas), UL, HRHRC Collection, HRC 55 \\
\hline 40 & 15720524 & Gérard du Vivier & Abraham Ortelius & Den Haag, KB, MS 79 C 4 (032), fol. 67 \\
\hline 41 & [1572] 0831 & William Soone & Abraham Ortelius & Den Haag, KB, MS 79 C 4 (033), fol. 209-210 \\
\hline 42 & 15721020 & Daniel Rogers & Abraham Ortelius & Austin (Texas), UL, HRHRC Collection, HRC 55 \\
\hline 43 & {$\left[\begin{array}{llllllllllll}1573 & 00 & 00\end{array}\right]$} & Nicholas Reynolds & Abraham Ortelius & London, BL, Add. MS 63650 Q (= fol. 59) \\
\hline 44 & 15730902 & Joannes Sambucus & Abraham Ortelius & Brussel, KB, MS III 936, nr. 25 \\
\hline 45 & 15740221 & Hubertus Goltzius & Abraham Ortelius & Den Haag, KB, MS 79 C 4 (021), fol. 101 \\
\hline 46 & 15740308 & Hiob Magdeburg & Abraham Ortelius & Austin (Texas), UL, HRHRC Collection, HRC 55 \\
\hline 47 & {$\left[\begin{array}{llllllllllllllllllllllll}1574 & 00 & 00\end{array}\right]$} & Victor Giselinus & Abraham Ortelius & Den Haag, KB, MS 79 C 4 (034), fol. 98 \\
\hline 48 & 15740420 & Victor Giselinus & Abraham Ortelius & Den Haag, KB, MS 79 C 4 (035), fol. 99 \\
\hline 49 & 15740426 & Cesare Orlandi & Abraham Ortelius & Austin (Texas), UL, HRHRC Collection, HRC 55 \\
\hline 50 & 15740531 & Abraham Ortelius & Jacobus Colius senior & Brussel, KB, MS III 936, nr. 4 \\
\hline 51 & 15740731 & Joannes Castelius & Abraham Ortelius & Den Haag, KB, MS 79 C 4 (036), fol. 36 \\
\hline 52 & 15740910 & Gabriel de Çayas & Abraham Ortelius & Den Haag, KB, MS 79 C 4 (037), fol. 37-38 \\
\hline
\end{tabular}




\begin{tabular}{l|l}
53 & 15741010 \\
54 & 15750205 \\
55 & 15750325 \\
56 & 15750402 \\
57 & 15750525 \\
58 & 15750605 \\
59 & 15751004 \\
60 & 15751021 \\
61 & 15751114 \\
62 & 15760228 \\
63 & 15761106 \\
65 & 15770101 \\
67 & 15770116 \\
68 & {$[157710227$} \\
69 & 15770601 \\
70 & {$[157710603$} \\
71 & 15770804 \\
72 & 15770924 \\
73 & $1578 \quad 00] 25$ \\
74 & 15780325 \\
75 & 15780715 \\
76 & 15780819 \\
77 & 15781007 \\
78 & 15781024 \\
79 & 15781026 \\
80 & 15781231 \\
81 & $1579[0000]$ \\
82 & 15790225 \\
83 & 15790405 \\
84 & 15790515 \\
85 & 15790701 \\
86 & {$[1579] 0711$} \\
87 & 15790711 \\
88 & 15790722 \\
89 & 15791015 \\
90 & 15791030 \\
91 & 15791101 \\
92 & 15800116 \\
93 & 15800202 \\
94 & 15800513 \\
95 & 15800730 \\
96 & 15800804 \\
97 & 15800815 \\
98 & 15801110 \\
99 & 15801212 \\
00 & 15801224 \\
01 & 15801229 \\
03 & 15810401 \\
04 & 15810424 \\
05 & 15810622 \\
06 & 15810630 \\
07 & 15810710 \\
108 & {$[158110717$} \\
& 1510 \\
\hline
\end{tabular}

\section{Melchior Lorichs}

Willem Canter

Cornelius Gemma

Abraham Ortelius

Johann Crato von Crafftheim

Joannes Isaac Levita

Jean Matal

Arnold Wachtendonck

Benito Arias Montano

Hieronymus Scholiers

Joannes I Moretus

John Dee

Peter Heyns

Alexander Grapheus

Joachim Camerarius junior

William Camden

William Camden

Herman Hortenberg

Paulus Melissus

Dirck Volckertsz. Coornhert

Daniel Rogers

Joannes Antonius Barvicius

William Camden

Johann Crato von Crafftheim

Alexander Grapheus

Guillaume Postel

Daniel Printz

Alexander Grapheus

Giovanni Federico Madruzzo

Francesco Soranzo

Joannes Gevae
Joannes Moflin

Lambertus à Bommeln

Jacobus Monavius

Johann Crato von Crafftheim

Nicolaus Secovius

Daniel Rogers

Daniel Engelhard
Clemens Friccius

Guido Laurinus

Georgius Braun

Petrus von Edeling

Arnoldus Freitag

Gerardus Mercator

Egnazio Danti

Jean Matal

Martinus Lydius

Richard Mulcaster

Hubertus Goltzius

Jacobus Monavius

Petrus von Edeling

Abraham Ortelius
Abraham Ortelius

Abraham Ortelius

Abraham Ortelius

Abraham Ortelius

Jacobus Colius junior (Ortelianus)

Abraham Ortelius

Abraham Ortelius

Abraham Ortelius

Abraham Ortelius

Abraham Ortelius

Abraham Ortelius

Abraham Ortelius

Abraham Ortelius

Abraham Ortelius

Abraham Ortelius

Abraham Ortelius

Abraham Ortelius

Abraham Ortelius

Abraham Ortelius

Abraham Ortelius

Abraham Ortelius

Abraham Ortelius

Abraham Ortelius

Abraham Ortelius

Abraham Ortelius

Abraham Ortelius

Abraham Ortelius

Abraham Ortelius

Abraham Ortelius

Abraham Ortelius

Abraham Ortelius

Abraham Ortelius

Abraham Ortelius

Abraham Ortelius

Abraham Ortelius

Abraham Ortelius

Abraham Ortelius

Abrahan Ortelius

Abraham Ortelius

Abraham Ortelius

Abraham Ortelius

Abraham Ortelius

Abraham Ortelius

Abraham Ortelius

Abraham Ortelius

Abraham Ortelius

Abraham Ortelius

Abraham Ortelius

Abraham Ortelius

Abraham Ortelius

Abraham Ortelius

Abraham Ortelius

Abraham Ortelius

Abraham Ortelius

Jacobus Colius senior
Den Haag, KB, MS 79 C 4 (038), fol. 128

Den Haag, KB, MS 79 C 4 (039), fol. 31

Chapel Hill, University of North Carolina, UL

Den Haag, KB, MS 79 C 4 (040), fol. 90-91

Brussel, KB, MS III 936, nr. 5

Den Haag, KB, MS 79 C 4 (025), fol. 61

Den Haag, KB, MS 79 C 4 (041), fol. 119

Austin (Texas), UL, HRHRC Collection, HRC 55

Den Haag, KB, MS 79 C 4 (042), fol. 233

Austin (Texas), UL, HRHRC Collection, HRC 55

Den Haag, KB, MS 79 C 4 (043), fol. 202-203

Sotheby's 1968, lot 375: buyer Bernard H. Breslauer (London)

New York (NY), Pierpont Morgan Library, LHMS, Misc English, MA 2637

Den Haag, KB, MS 79 C 4 (044), fol. 114

Den Haag, KB, MS 79 C 4 (045), fol. 105-106

Not in auction catalogue Sotheby's 1968

New York (NY), Pierpont Morgan Library, LHMS, Misc English, MA 2635

Austin (Texas), UL, HRHRC Collection, HRC 55

Den Haag, KB, MS 79 C 4 (046), fol. 170

Den Haag, KB, MS 79 C 4 (048), fol. [198?-]199

Den Haag, KB, MS 79 C 4 (050), fol. 50

Los Angeles (Cal.), Dean Jean Phillipps

Austin (Texas), UL, HRHRC Collection, HRC 55

New York (NY), Pierpont Morgan Library, LHMS, Misc English, MA 2636

Den Haag, KB, MS 79 C 4 (026), fol. 62

Cambridge (Mass.), Harvard University, Houghton Library, MS Typ. 557 [included in Hessels 83!

Den Haag, KB, MS 79 C 4 (051), fol. 176-177

Den Haag, KB, MS 79 C 4 (052), fol. 178-179

Cambridge (Mass.), Harvard University, Houghton Library, MS Typ. 557

Den Haag, KB, MS 79 C 4 (053), fol. 135-136

Den Haag, KB, MS 79 C 4 (054), fol. 214

Den Haag, KB, MS 79 C 4 (056), fol. 92-93

Den Haag. KB, MS 79 C 4 (059), fol. 18

Austin (Texas), UL, HRHRC Collection, HRC 55

Den Haag, KB, MS 79 C 4 (027), fol. 63-64

Austin (Texas), UL, HRHRC Collection, HRC 55

Los Angeles (Cal.), Dean Jean Phillipps

Den Haag, KB, MS 79 C 4 (060), fol. $72-73$

Den Haag, KB, MS 79 C 4 (063), fol. 88-89

Den Haag, KB, MS 79 C 4 (004), fol. 125

Den Haag, KB, MS 79 C 4 (064), fol. 23-24

Den Haag, KB, MS 79 C 4 (065), fol. 69-70

Den Haag, KB, MS 79 C 4 (067), fol. 85

Washington (D.C.), Library of Congress, Kraus H.P. Sir Francis Drake, nr. 2a

Austin (Texas), UL, HRHRC Collection, HRC 55

Austin (Texas), UL, HRHRC Collection, HRC 55

Den Haag, KB, MS 79 C 4 (070), fol. 131

London, BL, Add. MS 64080

Den Haag, KB, MS 79 C 4 (022), fol. 102

Den Haag, KB, MS 79 C 4 (071), fol. 144

Den Haag, KB, MS 79 C 4 (066), fol. 71

Brussel, KB, MS III 936, nr. 6 


\begin{tabular}{l|l|l}
109 & 15810812 & Arnoldus Freitag \\
110 & 15810901 & Nicolaus III Rhedinger \\
111 & 15811215 & Hugo Donellus \\
112 & 15820120 & Herman Hortenberg \\
113 & 15820211 & Andreas Schott \\
114 & 15820401 & Andreas Dudith \\
115 & 15820602 & William Charke \\
116 & 15820620 & Nicolaus III Rhedinger \\
117 & $15821105^{*}$ & Adolphus III Occo \\
118 & $15821128 *$ & Nicolaus Fabri \\
119 & $15821202 *$ & Cornelius Aggerius \\
120 & $15821202 *$ & Matthaeus Hostus \\
121 & $15[830103 *$ & William Charke \\
122 & {$[1583 ? 0210$ (note 1) } & Marcus Laurinus junior \\
123 & $15830310 *$ & Paulus Melissus \\
124 & 15830401 & Nicolas Clément \\
125 & $15830522 *$ & Reiner Reineccius \\
126 & 15830625 & Johan Roma \\
127 & $15830910 *$ & Johann Crato von Crafftheim \\
128 & $15830920 *$ & Arnoldus Freitag \\
129 & 15830921 & Agostino Musto \\
130 & $15830928 *$ & Laurentius Michaelis (von Hohenkirchen) \\
131 & 15831217 & Bonaventura Vulcanius \\
132 & $15831223 *$ & Laurentius Michaelis (von Hohenkirchen) \\
134 & {$[15840000]$} & Anselmus Stöckel \\
135 & 15840101 & Emanuel Josephus Adorno \\
137 & 15840430 & Agostino Musto \\
138 & 15840610 & Pierre Rutz \\
139 & 15840618 \\
140 & $15840808 *$ & Joannes Moflin \\
141 & 15841107 & William Charke \\
142 & 15841122 & Francesco Soranzo \\
143 & {$[1585-1589] 0402$} & Abraham Ortelius \\
144 & 15860109 & André Thevet \\
145 & {$[15860131 *$} & Abraham Ortelius \\
146 & 15860624 & William Camden \\
147 & 15861010 & Andreas Schott \\
148 & 15861117 & Joannes Vincentius Porta \\
149 & 15870119 & Abraham Ortelius \\
150 & 15870606 & Abraham Ortelius \\
151 & 15870612 & Christophe Plantin \\
152 & $15870612 *$ & Christophe d'Assonleville \\
153 & $15870918 *$ & Thomas Penny \\
154 & 15871102 & Joannes Posthius \\
155 & 15880106 & Dominicus Lampsonius \\
156 & 15880212 & Joannes Hass \\
157 & 15880616 \\
159 & $15880824 *$ & Janus Gruterus \\
160 & 15880829 & Niccolò Antonio Stelliola \\
161 & 15880930 & Peter Heyns \\
162 & 15881129 & Joachim Camerarius junior \\
164 & 15890515 & Abraham Ortelius \\
167 & 15890920 & Simon de Grimaldi \\
& Abraham Ortelius \\
Jean Jacques Boissard \\
\end{tabular}

Abraham Ortelius

Abraham Ortelius

Abraham Ortelius

Abraham Ortelius

Abraham Ortelius

Abraham Ortelius

Abraham Ortelius

Abraham Ortelius

Abraham Ortelius

Abraham Ortelius

Abraham Ortelius

Abraham Ortelius

Abraham Ortelius

Abraham Ortelius

Abraham Ortelius

Abraham Ortelius

Abraham

Abraham Ortelius

Abraham Ortelius

Abraham Ortelius

Abraham Ortelius

Abraham Ortelius

Abraham Ortelius

Abraham Ortelius

Abraham Ortelius

Abraham Ortelius

Abraham Ortelius

Abraham Ortelius

Abraham Ortelius

Abraham Ortelius

Emanuel van Meteren

Abraham Ortelius

Jacobus Colius junior (Ortelianus)

Abraham Ortelius

Abraham Ortelius

Abraham Ortelius

Abraham Ortelius

Emanue van Meteren

Jacobus Colius jun

Abraham Ortelius
Abraham Ortelius

Abraham Ortelius

Abraham Ortelius

Abraham Ortelius

Abraham Ortelius

Abraham Ortelius

Abraham Ortelius

Abraham Ortelius

Abraham Ortelius

Jacobus Colius senior

Abraham Ortelius

Jacobus Colius junior (Ortelianus)

Abraham Ortelius
Den Haag, KB, MS 79 C 4 (068), fol. 86

Den Haag, KB, MS 79 C 4 (072), fol. 190-191

Den Haag, KB, MS 79 C 4 (074), fol. 66

Den Haag, KB, MS 79 C 4 (047), fol. 171

Den Haag, KB, MS 79 C 4 (076), fol. 205

Den Haag, KB, MS 79 C 4 (075), fol. 68

Den Haag, KB, MS 79 C 4 (079), fol. 39

Den Haag, KB, MS 79 C 4 (073), fol. 192

Austin (Texas), UL, HRHRC Collection, HRC 55

Den Haag, KB, MS 79 C 4 (082), fol. 77

Den Haag, KB, MS 79 C 4 (083), fol. 3

Den Haag, KB, MS 79 C 4 (084), fol. 118

Den Haag, KB, MS 79 C 4 (080), fol. 40

Den Haag, KB, MS 79 C 4 (049), fol. 200-201

Den Haag. KB, MS 79 C 4 (085), fol. 43-45

Den Hag, KB, MS 79 C 4 (009), fol. 186-187

Austin (Texas), UL, HRHRC Collection, HRC 55

Den Haag, KB, MS 79 C 4 (028), fol. 65

Den Haag, KB, MS 79 C 4 (069), fol. 87

Den Haag, KB, MS 79 4 (011), fol. 154-155

Austin (Texas), UL, HRHRC Collection, HRC 55

Leiden, UB, MS BPL 2766

Den Haag, KB, MS 79 C 4 (013), fol. 143

Den Haag, KB, MS 79 C 4 (014), fol. 217

Den Haag, KB, MS 79 C 4 (015), fol. 1-2

Den Haag, KB, MS 79 C 4 (012), fol. 156-157

Den Haag, KB, MS 79 C 4 (016), fol. 195-196

Den Haag, KB, MS 79 C 4 (058), fol. 160-161

Den Haag, KB, MS 79 C 4 (081), fol. 41

Den Haag, KB, MS 79 C 4 (055), fol. 215

Paris, Institut Néerlandais, Fondation Custodia, 1974-A.121

Not in auction catalogue Soth

KB, MS III 936, nr 7

Not in auction catalogue Sotheby's 1968

Den Hal 206

Cambridge (Mass.), Harvard University, Houghton Library, MS Lat. 225 (1)

ry, LHMS, Unbound International Ortelius, MA 2634

Cambridge (Mass.), Harvard University, Houghton Library, MS Lat. 225 (2)

Austin (Texas), UL, HRHRC Collection, HRC 55

Den Haag, KB, MS 79 C 4 (092), fol. 172

Den Haag, KB, MS 79 C 4 (093), fol. 178-179

Den Haag, KB, MS 79 C 4 (094), fol. 121-122

Den Haag, KB, MS 79 C 4 (095), fol. 112-113

Sotheby's 1968, lot 360: buyer Hofmann 8 Freeman (Sevenoaks)

Den Haag, KB, MS 79 C 4 (096), fol. 216

Den Haag, KB, MS 79 C 4 (098), fol. 115

Not in auction catalogue Sotheby's 1968

Brussel, KB, MS III 936, nr. 8

Den Haag, KB, MS 79 C 4 (099), fol. 108-109

Not in auction catalogue Sotheby's 1968 
Janus Gruterus

Joachim Camerarius junio

Hilps van Winghe

Abraham Ortelius

Richard Hakluyt senior

Benito Arias Montano

Abraham Ortelius

Friedrich Sylburg

Dominicus Lampsonius

Benito Arias Montano

Joannes Jonas Iavilc Moravus

Hieronymus Commelinus

Thomas van Rye

Hieronymus Megiser

Abronymus Megiser

Filips van Winghe

Filips van Winghe

Carolus Brooman

Carolus Broom
Justus Lipsius

Franciscus I Raphelengius

Justus Lipsius

Jacobus Colius junior (Ortelianus)

Jean Jacques Boissard

Benito Arias Montan

Abraham Ortelius

Carolus Clusius

Justus Lipsius

Abraham Ortelius

Filippo Pigafetta

Justus Lipsius

Dionysius de Villers

Hieronymus Commelinus

Marcus Welser

Justus Lipsius

Joannes Radermacher senior

Carolus Clusius

Justus Lipsius

Luis Teixeira

Petrus Verheyl

Abraham Ortelius

Friedrich Sylburg

Abraham Ortelius

Dionysius de Villers

Franciscus I Raphelengius

Filips van Winghe

Abraham Ortelius

Melchior Moretus

Bonaventura Vulcanius

Franciscus I Raphelengius

Jan Bernaerts

Hieronymus van Winghe

Joannes Baptista Faviola
Abraham Orteliu

Abraham Ortelius

Abraham Ortelius

Dominicus Lampsonius

Abraham Ortelius

Abraham Ortelius

Emanuel van Meteren

Abraham Ortelius

Abraham Ortelius

Abraham Ortelius

Abraham Ortelius

Abraham Ortelius

Abraham Ortelius

Abraham Ortelius

Jacobus Colius junior (Ortelianus)

Abraham Ortelius

Abraham Ortelius

Abraham Ortelius

Abraham Ortelius

Abraham Ortelius

Abraham Ortelius

Abraham Ortelius

Abraham Ortelius

Abraham Ortelius

Jacobus Colius junior (Ortelianus)

Abraham Ortelius

Abraham Ortelius

Jacobus Colius junior (Ortelianus)

Abraham Ortelius

Abraham Ortelius

Abraham Ortelius

Abraham Ortelius

Abraham 0

Abraham Ortelius

Jacobus Colius junior (Ortelianus)

Abraham Ortelius

Jacobus Colius junior (Ortelianus)

Abraham Ortelius

Abraham Ortelius

Abraham Ortelius

Emanuel van Meteren

Abraham Ortelius

Abraham Ortelius

Abraham Ortelius

Abraham Ortelius

Abraham Ortelius

Abraham Ortelius
Sotheby's 1968, lot 360: buyer Hofmann \& Freeman (Sevenoaks

logue Sotheby's 1968

Leiden, UB, MS BPL 2766

Brussel, KB, MS III 936, nr. 9

Austin (Texas), UL, HRHRC Collection, HRC 55

Austin (Texas), UL, HRHRC Collection, HRC 55

Brussel, KB, MS III 936, nr. 10

Den Haag, KB, MS 79 C 4 (103), fol. 218

Brussel, KB, MS III 936, nr. 9 [in the margins of Hessels 171!]

Austin (Texas), UL, HRHRC Collection, HRC 55

Austin (Texas), UL, HRHRC Collection, HRC 55

Den Haag, KB, MS 79 C 4 (106), fol. 46

Den Haag, KB, MS 79 C 4 (108), fol. 197

Den Haag, KB, MS 79 C 4 (104), fol. 219

Austin (TexB, MS 79 C 4 (109), fol. 140-141

Leiden, UB, MS BPL 2766

Den Haag, KB, MS 79 C 4 (110), fol. 26-27

Den Haag, KB, MS 79 C 4 (111), fol. 28

Brussel, KB, MS III 936, nr. 15

Sotheby's 1968, lot 384: buyer Nico Israel (Amsterdam)

Brussel, KB, MS III 936, nr. 16

Den Haag, KB, MS 79 C 4 (112), fol. 48

Den Haag, KB, MS 79 C 4 (101), fol. 15-16

Austin (Texas), UL, HRHRC Collection, HRC 55

Paris, Institut Néerlandais, Fondation Custodia, 1972-A.3

Not in auction catalogue Sotheby's 1968

Brussel, KB, MS III 936, nr. 17

Brussel, KB, MS III 936, nr. 11

Austin (Texas), UL, HRHRC Collection, HRC 55

Brussel, KB, MS III 936, nr. 18

Den Haag, KB, MS 79 C 4 (114), fol. 227
Den Haag, KB, MS 79 C 4 (107), fol. 47

New York (NY), Pierpont Morgan Library, LHMS, Unbound International Welser, MA 2638 (1)

Brussel, KB, MS III 936, nr. 19

Den Haag, KB, MS 79 C 4 (019), fol. 184

Not in auction catalogue Sotheby's 1968

Brussel, KB, MS III 936, nr. 20

Den Haag, KB, MS 79 C 4 (116), fol. 221-222

Den Haag, KB, MS 79 C 4 (117), fol. 226

Den Haag, KB, MS 79 C 4 (118), fol. 167

Den Haag, KB, MS 79 C 4 (105), fol. 22

Brussel, KB, MS III 936, nr. 12

Den Haag, KB, MS 79 C 4 (115), fol. 228-229

Sotheby's 1968, lot 384: buyer Nico Israel (Amsterdam)

Leiden, UB, MS BPL 2766

Brussel, KB, MS III 936, nr. 13

Den Haag, KB, MS 79 C 4 (119), fol. 152-153

Leiden, UB, MS BPL 2766

Sotheby's 1968, lot 384: buyer Nico Israel (Amsterdam)

Den Haag, KB, MS 79 C 4 (120), fol. 10-11

Den Hag, KB, MS 79 C 4 (089), fol. 234 


\begin{tabular}{l|l|l}
225 & 15921124 (note 2) & Emanuel van Meteren \\
226 & 15921206 & Franciscus I Raphelengius \\
227 & 15930109 & Angelo Breventano \\
228 & 15930127 & Abraham Ortelius \\
229 & {$[15930300]$} & Abraham Ortelius \\
230 & 15930305 & Franciscus II Raphelengius \\
231 & $15930408 *$ & Carolus Clusius \\
232 & 15930416 & Joannes Baptista Faviola \\
233 & 15930504 & Jean Jacques Boissard \\
234 & 15930613 & Pieter Bert \\
237 & 15930725 (note 3) & Carolus Clusius \\
238 & $15930818 *$ & Nathan Chytraeus \\
239 & 15930920 & Joris Hoefnagel \\
240 & 15930926 & Manuel Barbosa \\
241 & 15940131 & Claes Govaertsz. \\
242 & 15940215 & Jacobus Monavius \\
243 & 15940328 & Joannes I Sadeler and Raphael I Sadeler \\
244 & 15940427 & Franciscus I Raphelengius \\
245 & $15940504 *$ & John Johnston \\
246 & 15940509 & Laurens van Goidtsenhoven (van Haecht) \\
247 & 15940514 & Jean L'Heureux \\
248 & 15940620 & Joannes Vivianus \\
249 & 15940803 & Janus Gruterus \\
250 & 15940812 & Franciscus I Raphelengius \\
251 & 15940820 & Gaspar Alvares Louzada Machado \\
252 & 15940901 & Georgius Fleck \\
253 & $15940919 *$ & Reiner Reineccius \\
254 & 15940926 & Andreas Schott \\
255 & 15941008 & Jacques de Carondelet \\
256 & 15941011 & Mello Brunsema \\
257 & 15941107 & Nicolaus de Vrise \\
258 & 15941201 & Manuel Barbosa \\
259 & 15941226 & Arnoldus Mylius \\
260 & {$[15940000]$} & Louis d'Orléans \\
261 & 15950104 & Abraham Ortelius \\
262 & 15950119 (note 4) & Emanuel van Meteren \\
263 & 15950122 & Georgius Braun \\
264 & 15950202 & Joannes Murdisonus \\
265 & 15950202 & Abraham Ortelius \\
268 & 15950303 & \\
269 & 15950318 & Claude Brulart \\
270 & 15950427 & Jean L'Heureux \\
271 & 15950617 & Gerard van Corck \\
272 & 15950617 & Paulus Merula \\
273 & 15950706 & Joannes Jonas Iavilc Moravus \\
275 & 15950827 & Georgius ab Austria junior \\
276 & 15950905 & Friedrich Lindenbrog \\
277 & 15950921 & Georgius Fleck \\
278 & 15951018 & Justus Lipsius \\
279 & 15951027 & Abraham Ortelius \\
280 & {$[1595] 1114$} & Adam de la Planche \\
281 & 15951228 & Friedrich Lindenbrog \\
282 & 15960201 & Jaques Marchant \\
& & Joannes Woverius \\
\hline &
\end{tabular}

Abraham Ortelius

Abraham Ortelius

Abraham Ortelius

Jacobus Colius junior (Ortelianus)

Jacobus Colius junior (Ortelianus)

Abraham Ortelius

Abraham Ortelius

Abraham Ortelius

Abraham Ortelius

Abraham Ortelius

Abraham Ortelius

Abraham Ortelius

Abraham Ortelius

Abraham Ortelius

Abraham Ortelius

Abraham Ortelius

Abraham Ortelius

Abraham Ortelius

Abraham Ortelius

Abraham Ortelius

Abraham Ortelius

Abraham Ortelius

Abraham Ortelius

Abraham Ortelius

Abraham Ortelius

Abraham Ortelius

Abraham Ortelius

Abraham Ortelius

Abraham Ortelius

Abraham Ortelius

Abraham Ortelius

Abraham Ortelius

Abraham Ortelius

Abraham Ortelius

Aacobus Colius junior (Ortelianus)

Abraham Ortelius

Abraham Ortelius

Abraham Ortelius

Jacobus Colius junior (Ortelianus)

\section{Abraham Ortelius}

Abraham Ortelius

Abraham Ortelius

Abraham Ortelius

Abraham Ortelius

Abraham Ortelius

Abraham Ortelius

Abraham Ortelius

Abraham Ortelius

Jacobus Colius junior (Ortelianus)

Abraham Ortelius

Abraham Ortelius

Abraham Ortelius

Abraham Ortelius
Brussel, KB, MS III 936, nr. 2

Sotheby's 1968, lot 384: buyer Nico Israel (Amsterdam)

Den Haag, KB, MS 79 C 4 (123), fol. 25

Sotheby's 1968, lot 337. buyer Kenneth Mummery (Bournemouth

Sotheby's 1968, lot 338: buyer Kenneth Mummery (Bournemouth)

Sotheby's 1968, lot 384: buyer Nico Israel (Amsterdam)

Not in auction catalogue Sotheby's 1968

Den Haag, KB, MS 79 C 4 (122), fol. 79

Den Haag, KB, MS 79 C 4 (102), fol. 17

Den Haag, KB, MS 79 C 4 (124), fol. 12

Not in auction catalogue Sotheby's 1968

Den Haag, KB, MS 79 C 4 (125), fol. 42

\section{Not in auction catalogue Sotheby's 1968}

Austin (Texas), UL, HRHRC Collection. HRC 55

Den Haag, KB, MS 79 C 4 (126), fol. 103-104

Den Haag KB, MS 79 C 4 (127), fol. 145 [-146?)

Den Haag, KB, MS 79 C 4 (127), fol. 145[-146?]

Sotheby's 1968, lot 384: buyer Nico Israel (Amsterdam)

St Andrews, UL, MS 30458

Den Haag, KB, MS 79 C 4 (128), fol. 110-111

Den Haag, KB, MS 79 C 4 (086), fol. 132

Austin (Texas), UL, HRHRC Collection, HRC 55

Sotheby's 1968, lot 360: buyer Hofmann \& Freeman (Sevenoaks)

Sotheby's 1968, lot 384: buyer Nico Israel (Amsterdam)

Den Haag, KB, MS 79 C 4 (129), fol. 129-130

Den Haag, KB, MS 79 C 4 (130), fol. 80-81

Den Haag, KB, MS 79 C 4 (010), fol. 188-189

Den Haag, KB, MS 79 C 4 (078), fol. 207-208

Den Haag, KB, MS 79 C 4 (132), fol. 32-33

Den Haag, KB, MS 79 C 4 (134), fol. 30

Den Haag, KB, MS 79 C 4 (090), fol. 232

Austin (Texas), UL, HRHRC Collection, HRC 55

Austin (Texas), UL, HRHRC Collection, HRC 55

Den Haag KB, MS 79 C 4 (135), fol. 166

Sotheby's 1968, lot 339: buyer Nico Israel (Amsterdam)

Brussel, KB, MS III 936, nr. 24

Sotheby's 1968, lot 350: buyer Nico Israel (Amsterdam)

St Andrews, UL, MS 30459

Sotheby's 1968, lot 340: buyer Kenneth Mummery (Bournemouth): auctioned at Christie's London, 3

June 2009, sale 7725, lot 38; auctioned at Stargardt Berlin, 5 June 2012, Katalog 698, lot 418

Den Haag, KB, MS 79 C 4 (136), fol. 29

Den Haag, KB, MS 79 C $4(087)$, fol. 133

Den Haag, KB, MS 79 C $4(137)$, Kol. $53-58$

Den Haag, KB, MS 79 C 4 (138), fol. 142

Den Haag, KB, MS 79 C 4 (139), fol. 150-1

Den Haag, KB, MS 79 C 4 (140), fol. 8-9

Den Haag, KB, MS 79 C 4 (141), fol. 126

Den Haag, KB, MS 79 C 4 (131), fol. 82

Brussel, KB, MS III 936, nr. 21

Sotheby's 1968, lot 341: buyer Kenneth Mummery (Bournemouth)

Austin (Texas), UL, HRHRC Collection, HRC 55

Den Haag, KB, MS 79 C 4 (142), fol. 127

Den Haag, KB, MS 79 C 4 (143), fol. 138-139

Den Haag, KB, MS 79 C 4 (144), fol. 235 


\begin{tabular}{|c|c|c|c|c|}
\hline 283 & 15960225 & Charles de Ligne, prince-count of Arenberg & Abraham Ortelius & Den Haag, KB, MS 79 C 4 (146), fol. 4-5 \\
\hline 284 & 15960312 & Paulus Monelia & Abraham Ortelius & Den Haag, KB, MS 79 C 4 (148), fol. 147-148 \\
\hline 285 & 15960322 & Bernardus Paludanus & Abraham Ortelius & Minneapolis (Minn.), UL, James Ford Bell Library, 1596 fPa \\
\hline 286 & 15960323 & Abraham Ortelius & Jacobus Colius junior (Ortelianus) & Den Haag, KB, MS 79 C 4 (091), fol. 168 \\
\hline 287 & 15960326 & Rumoldus Mercator & Abraham Ortelius & Brussel, KB, MS III 936, nr. 22 \\
\hline 288 & 15960426 & Benito Arias Montano & Abraham Ortelius & Austin (Texas), UL, HRHRC Collection, HRC 55 \\
\hline 289 & 15960427 & Petrus de Grassis & Abraham Ortelius & Den Haag, KB, MS 79 C 4 (151), fol. 107 \\
\hline 290 & 15960624 & Paulus Monelia & Abraham Ortelius & Den Haag, KB, MS 79 C 4 (149), fol. 149 \\
\hline 291 & 15960810 & Johann Georg von Werdenstein & Abraham Ortelius & Austin (Texas), UL, HRHRC Collection, HRC 55 \\
\hline 292 & 15960905 & Joannes Vivianus & Abraham Ortelius & Den Haag, KB, MS 79 C 4 (152), fol. 230 \\
\hline 293 & 15960921 & Jacques de Carondelet & Abraham Ortelius & Den Haag, KB, MS 79 C 4 (133), fol. 34-35 \\
\hline 294 & 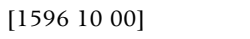 & Jacobus Colius junior (Ortelianus) & Abraham Ortelius & Den Haag, KB, MS 79 C 4 (113), fol. 49 \\
\hline 295 & 15961014 & Arnoldus Mylius & Abraham Ortelius & Den Haag, KB, MS 79 C 4 (154), fol. 162 \\
\hline 296 & 15961025 & Andreas Dycchius & Abraham Ortelius & Austin (Texas), UL, HRHRC Collection, HRC 55 \\
\hline 297 & 15961213 & Daniel Engelhard & Abraham Ortelius & Den Haag, KB, MS 79 C 4 (061), fol. 74-75 \\
\hline 298 & 15961220 & Bernardus Paludanus & Abraham Ortelius & Minneapolis (Minn.), UL, James Ford Bell Library, 1596 fPa \\
\hline 299 & 15961225 & Caspar Schoppe & Abraham Ortelius & Den Haag, KB, MS 79 C 4 (155), fol. 204 \\
\hline 300 & 15961231 & Bonaventura Vulcanius & Abraham Ortelius & Leiden, UB, MS BPL 2766 \\
\hline 302 & 15970402 * & Tobias Scultetus & Abraham Ortelius & Den Haag, KB, MS 79 C 4 (156), fol. 211-212 \\
\hline 303 & 15970403 & Abraham Ortelius & Jacobus Colius junior (Ortelianus) & Sotheby's 1968, lot 343: buyer Kenneth Mummery (Bournemouth) \\
\hline 304 & 15970606 & Joachim Camerarius junior & Abraham Ortelius & Not in auction catalogue Sotheby's 1968 \\
\hline 305 & 15970625 & Carolus Clusius & Abraham Ortelius & Not in auction catalogue Sotheby's 1968 \\
\hline 306 & 15970702 & Marcus Welser & Abraham Ortelius & New York (NY), Pierpont Morgan Library, LHMS, Unbound International Welser, MA 2638 (2) \\
\hline 307 & 15970813 & Joannes Maior & Abraham Ortelius & Den Haag, KB, MS 79 C 4 (157), fol. 137 \\
\hline 308 & 15970828 & Joannes Vivianus & Abraham Ortelius & Den Haag, KB, MS 79 C 4 (153), fol. 231 \\
\hline 309 & 15971018 & Jacobus Colius junior (Ortelianus) & Abraham Ortelius & Austin (Texas), UL, HRHRC Collection, HRC 55 \\
\hline 310 & 15971025 & Jean L'Heureux & Abraham Ortelius & Den Haag, KB, MS 79 C 4 (088), fol. 134 \\
\hline 311 & 15971113 & David Hoeschel & Abraham Ortelius & Den Haag, KB, MS 79 C 4 (158), fol. 116 \\
\hline 312 & 15971121 & David Hoeschel & Abraham Ortelius & Den Haag, KB, MS 79 C 4 (159), fol. 117 \\
\hline 313 & 15971201 & Marquard Friedrich Freher & Abraham Ortelius & Den Haag, KB, MS 79 C 4 (163), fol. 83-84 \\
\hline 314 & 15980124 & Abraham Ortelius & Jacobus Colius junior (Ortelianus) & Den Haag, KB, MS 79 C 4 (150), fol. 169 \\
\hline 315 & 15980213 & Charles de Ligne, prince-count of Arenberg & Abraham Ortelius & Den Haag, KB, MS 79 C 4 (147), fol. 6-7 \\
\hline 316 & 15980214 & Bonaventura Vulcanius & Abraham Ortelius & Leiden, UB, MS BPL 2766 \\
\hline 317 & 15980323 & Daniel Engelhard & Abraham Ortelius & Den Haag, KB, MS 79 C 4 (062), fol. 76 \\
\hline 318 & 15980404 & Joannes Woverius & Abraham Ortelius & Den Haag, KB, MS 79 C 4 (145), fol. 236 \\
\hline 319 & 15980422 & Henricus Sedulius & Abraham Ortelius & Den Haag, KB, MS 79 C 4 (162), fol. 213 \\
\hline 320 & 15980504 * & Adolphus III Occo & Abraham Ortelius & Den Haag, KB, MS 79 C 4 (161), fol. 165 \\
\hline 322 & 15980603 & Abraham Ortelius & Jacobus Colius junior (Ortelianus) & Brussel, KB, MS III 936, nr. 14 \\
\hline 372 & [0000] 1016 (note 5) & William Rainolds & Abraham Ortelius & Den Haag, KB, MS 79 C 4 (160), fol. 185 \\
\hline 376 & 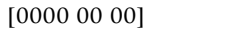 & Jacobus Philippus de Bisschop & Abraham Ortelius & Gent, UL, MS 3618 \\
\hline
\end{tabular}

* Presumably dated in Old Style (Julian calendar)

(note 1) Hessels dated the letter presumably in 1583. J. Smits, 'Een dankbrief, 19 jaar na dato?', De gulden passer, 54 (1976), $72-76$ argues that the letter should be dated in 1565 . The Royal Library in Brussels, however, dates the letter in [1571] in the ann

(note 2) Dated 14/24 November 1592: 14 as Old Style (Julian calendar), 24 as New Style (Gregorian calendar

(note 3) Dated 15/25 July 1593: 15 as Old Style (Julian calendar), 25 as New Style (Gregorian calendar)

(note 4) Dated 9/19 January 1595: 9 as Old Style (Julian calendar), 19 as New Style (Gregorian calendar)

(note 5) To be dated between 1575 and 1592 
1.2. Letters to Jacobus Colius Ortelianus (excluding those from Ortelius)

\begin{tabular}{|c|c|c|c|c|}
\hline Hessels & Date & From & To & Autograph letter \\
\hline 158 & 15880713 & Joannes Lamoot & \begin{tabular}{|l|} 
Jacobus Colius junior (Ortelianus) \\
\end{tabular} & Den Haag, KB, MS 79 C 4 (097), fol. 120 \\
\hline 165 & 15890708 & Franciscus II Raphelengius & Jacobus Colius junior (Ortelianus) & Cambridge (Mass.), Harvard University, Houghton Library, MS Lat. 225 (4) \\
\hline 193 & 15910201 & Franciscus II Raphelengius & Jacobus Colius junior (Ortelianus) & Cambridge (Mass.), Harvard University, Houghton Library, MS Lat. 225 (4) \\
\hline 209 & 15920215 & Dirick Herweyers & Jacobus Colius junior (Ortelianus) & Leiden, MS BPL 2755 \\
\hline 236 & 15930701 & Petrus Montanus & Jacobus Colius junior (Ortelianus) & Leiden, MS BPL 2755 \\
\hline 266 & 15950214 & Jacobus Monavius & Jacobus Colius junior (Ortelianus) & Leiden, MS BPL 2755 \\
\hline 301 & 15970000 & Hieronymus Scholiers & Jacobus Colius junior (Ortelianus) & Leiden, MS BPL 2755 \\
\hline 321 & 15980601 & Marcus Welser & Jacobus Colius junior (Ortelianus) & Leiden, MS BPL 2755 \\
\hline 324 & 15990216 (note 6) & Adolphus III Occo & Jacobus Colius junior (Ortelianus) & Leiden, MS BPL 2755 \\
\hline 325 & 16010413 & Abraham van Goorle (Gorlaeus) & Jacobus Colius junior (Ortelianus) & Leiden, MS BPL 2755 \\
\hline 326 & 16020911 & Franciscus Sweertius junior & Jacobus Colius junior (Ortelianus) & Leiden, MS BPL 2755 \\
\hline 327 & 16021008 & Nicolaus Rockox & Jacobus Colius junior (Ortelianus) & Leiden, MS BPL 2755 \\
\hline 328 & 16030102 * & Jacobus Gruterus & Jacobus Colius junior (Ortelianus) & Leiden, MS BPL 2755 \\
\hline 329 & 16030702 & Joannes Woverius & Jacobus Colius junior (Ortelianus) & Leiden, MS BPL 2755 \\
\hline 330 & 16030725 & Joannes Radermacher senior & Jacobus Colius junior (Ortelianus) & Austin (Texas), UL, HRHRC Collection, HRC 55 \\
\hline 331 & 16030814 & Joannes Radermacher senior & Jacobus Colius junior (Ortelianus) & Austin (Texas), UL, HRHRC Collection, HRC 55 \\
\hline 332 & 16031130 & Joannes Woverius & Jacobus Colius junior (Ortelianus) & Leiden, MS BPL 2755 \\
\hline 333 & 16030000 & Joannes Brovaert & Jacobus Colius junior (Ortelianus) & Leiden, MS BPL 2755 \\
\hline 334 & 16040117 (note 7) & Joannes Radermacher senior & Jacobus Colius junior (Ortelianus) & Austin (Texas), UL, HRHRC Collection, HRC 55 \\
\hline 335 & 16060805 & Joannes Radermacher senior & Jacobus Colius junior (Ortelianus) & Austin (Texas), UL, HRHRC Collection, HRC 55 \\
\hline 336 & 16061216 & Franciscus II Raphelengius & Jacobus Colius junior (Ortelianus) & Leiden, MS BPL 2755 \\
\hline 337 & 16061216 * & Jean François le Petit & Jacobus Colius junior (Ortelianus) & Leiden, MS BPL 2755 \\
\hline 338 & 16070301 & Joannes Radermacher senior & Jacobus Colius junior (Ortelianus) & Austin (Texas), UL, HRHRC Collection, HRC 55 \\
\hline 339 & 16070620 & Franciscus Sweertius junior & Jacobus Colius junior (Ortelianus) & Leiden, MS BPL 2755 \\
\hline 340 & 16070915 * & Joannes Regius & Jacobus Colius junior (Ortelianus) & Leiden, MS BPL 2755 \\
\hline 341 & 16070924 & Jan Cole & Jacobus Colius junior (Ortelianus) & Leiden, MS BPL 2755 \\
\hline 342 & 16071008 & Dionysius de Villers & Jacobus Colius junior (Ortelianus) & Leiden, MS BPL 2755 \\
\hline 343 & 16071008 * & Paul de L'Obel & Jacobus Colius junior (Ortelianus) & Leiden, MS BPL 2755 \\
\hline 344 & 16071009 & Matthias de L'Obel junior & Jacobus Colius junior (Ortelianus) & Leiden, MS BPL 2755 \\
\hline 345 & 16071104 * & Pieter Cole & Jacobus Colius junior (Ortelianus) & Leiden, MS BPL 2755 \\
\hline 346 & {$\left[\begin{array}{lllllllllll}1608 & 00 & 00\end{array}\right]$} & Franciscus II Raphelengius & Jacobus Colius junior (Ortelianus) & Cambridge (Mass.), Harvard University, Houghton Library, MS Lat. 225 (3) \\
\hline 347 & 16080423 & Jan Vekemans & Jacobus Colius junior (Ortelianus) & Leiden, MS BPL 2755 \\
\hline 348 & 16090203 & Nicolas Claude Fabri de Peiresc & Jacobus Colius junior (Ortelianus) & Leiden, MS BPL 2755 \\
\hline 349 & 16090318 & David Hoeschel & Jacobus Colius junior (Ortelianus) & Leiden, MS BPL 2755 \\
\hline 350 & 16090402 & Johann Ulrich Rumler & Jacobus Colius junior (Ortelianus) & Leiden, MS BPL 2755 \\
\hline 351 & 16090805 & Aubertus Miraeus & Jacobus Colius junior (Ortelianus) & Leiden, MS BPL 2755 \\
\hline 352 & 16090910 * & Matthias de L'Obel senior & Jacobus Colius junior (Ortelianus) & Leiden, MS BPL 2755 \\
\hline 353 & 16100607 * & Matthias de L'Obel senior & Jacobus Colius junior (Ortelianus) & Leiden, MS BPL 2755 \\
\hline 354 & 16100917 & David Hoeschel & Jacobus Colius junior (Ortelianus) & Leiden, MS BPL 2755 \\
\hline 355 & 16101008 & Johann Ulrich Rumler & Jacobus Colius junior (Ortelianus) & Leiden, MS BPL 2755 \\
\hline 356 & 16120131 * & Alexander Rhedus & Jacobus Colius junior (Ortelianus) & Leiden, MS BPL 2755 \\
\hline 358 & 16121018 & A. de Bruyn & Jacobus Colius junior (Ortelianus) & Leiden, MS BPL 2755 \\
\hline 359 & 16130612 & Ottavio Pisani & Jacobus Colius junior (Ortelianus) & Leiden, MS BPL 2755 \\
\hline 360 & 16130624 & Ottavio Pisani & Jacobus Colius junior (Ortelianus) & Leiden, MS BPL 2755 \\
\hline 361 & 16131016 & Franciscus II Raphelengius & Jacobus Colius junior (Ortelianus) & Cambridge (Mass.), Harvard University, Houghton Library, MS Lat. 225 (4) \\
\hline 362 & 16171123 & Godefridus and Lazarus "medici Antverpienses" & Jacobus Colius junior (Ortelianus) & Leiden, MS BPL 2755 \\
\hline 364 & 16230127 & Abraham Bush & Jacobus Colius junior (Ortelianus) & Leiden, MS BPL 2755 \\
\hline 365 & 16230923 & Joannes Radermacher junior & Jacobus Colius junior (Ortelianus) & Leiden, MS BPL 2755 \\
\hline
\end{tabular}


Jacobus Colius junior (Ortelianus)

acobus Colius junior (Ortelianu

Leiden, MS BPL 2755

Leiden, MS BPL 2755

Leiden, MS BPL 2755

Leiden, MS BPL 2755

* Presumably dated in Old Style (Julian calendar)

(note 6) Dated 6/16 January 1599: 6 as Old Style (Julian calendar), 16 as New Style (Gregorian calendar) (note 7) Dated 7/17 January 1599: 7 as Old Style (Julian calendar), 17 as New Style (Gregorian calenda ) 
1.3. Other letters and documents

\begin{tabular}{|c|c|c|c|c|}
\hline Hessels & Date & From & To & Autograph letter \\
\hline 1 & 15240325 & Desiderius Erasmus & Guido Morillon & New York (NY), Pierpont Morgan Library, LHMS, Erasmus, MA 1620 \\
\hline 2 & 15241205 & Albrecht Dürer & Nicolaus Kratzer & Not in auction catalogue Sotheby's 1968 \\
\hline 3 & 15340830 & Desiderius Erasmus & Guido Morillon & New York (NY), Pierpont Morgan Library, LHMS, Erasmus, MA 1620 \\
\hline 4 & [1540] 0108 & Guillaume Budé & Guido Morillon & New York (NY), Pierpont Morgan Library, LHMS, Erasmus, MA 1621 \\
\hline 5 & 15490402 & Pierre Gilles & Jacques Amyot & Den Haag, KB, MS 79 C 4 (001), fol. 94-97 \\
\hline 12 & 15621122 & Jacobus Colius senior & Thomas, Mary, Christopher, Susanna and John Cole & Leiden, MS BPL 2766 \\
\hline 64 & 15641114 & Geeraert Janssen & Jacobus Colius senior & Not in auction catalogue Sotheby's 1968 \\
\hline 66 & 15770111 & Filips van Marnix van Sint-Aldegonde & Emanuel van Meteren & Not in auction catalogue Sotheby's 1968 \\
\hline 102 & 15810127 & Georgius Ratallerus & Franciscus I Raphelengius & Leiden, MS BPL 2766 \\
\hline 133 & 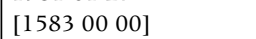 & Martinus Schilling & Jacobus Monavius & Leiden, MS BPL 2766 \\
\hline 136 & 15840429 & Filips van Marnix van Sint-Aldegonde & Emanuel van Meteren & Not in auction catalogue Sotheby's 1968 \\
\hline 163 & 15890228 & Dominicus Lampsonius & Louis de Montjosieu & Den Haag, KB, MS 79 C 5 \\
\hline 166 & 15890827 & Joannes Matthaeus Wacker von Wackenfels & [Jacobus Monavius?] & Leiden, MS BPL 2766 \\
\hline 183 & 15900802 & Fulvio Orsini & Nicolaus Rockox & Leiden, MS BPL 2766 \\
\hline 186 & 15900901 & Filips van Winghe & Dionysius de Villers & Leiden, MS BPL 2766 \\
\hline 235 & 15930614 & Justus Lipsius & Franciscus I Raphelengius & Sotheby's 1968, lot 384: buyer Nico Israel (Amsterdam) [cited in Hessels 244!] \\
\hline 267 & [1595 0220$]$ (note 8) & Laurens Beyerlinck & on the death of archduke Ernest & Leiden, MS BPL 2766 \\
\hline 274 & 15950801 & Joannes Matthaeus Wacker von Wackenfels & Jacobus Monavius & Leiden, MS BPL 2766 \\
\hline 323 & 15980707 & Filips Galle & Emanuel van Meteren & Leiden, MS BPL 2766 \\
\hline 357 & 16120408 & (note 9) & & Leiden, MS BPL 2766 \\
\hline 363 & 16200629 & $\begin{array}{l}\text { Thomas Morton (1564-1659, bishop of } \\
\text { Coventry and Lichfield) }\end{array}$ & $\begin{array}{l}\text { Marcus Antonius de Dominis (Dalmatian ecclesiastic, } \\
\text { apostate, and man of science) }\end{array}$ & Not in auction catalogue Sotheby's 1968 \\
\hline 369 & {$\left[\begin{array}{lllllllllll}1625 & 06 & 00\end{array}\right]$} & (note 10) & & Not in auction catalogue Sotheby's 1968 \\
\hline 373 & 00000000 & [Benito Arias Montano] & & Austin (Texas), UL, HRHRC Collection, HRC 55. [between 4 and 5] \\
\hline 374 & 00000000 & Josephus Justus Scaliger & & Paris, Institut Néerlandais, Fondation Custodia, 1970-A.208 \\
\hline 375 & 00000000 & Michael van der Haeghen & & Paris, Institut Néerlandais, Fondation Custodia, 1974-A.122 \\
\hline
\end{tabular}

(note 8) 20 February 1595 is the date of death of archduke Ernest. Laurens Beyerlinck (born 12 April 1578) was only 17 at the time.

(note 9) Epitaph on Emanuel van Meteren, who died 8 April 1612

(note 10) Description of a triumphal arch designed to be erected at the marriage of King Charles I with the Princess Henrietta Maria of France, by the foreign residents in London 